\title{
Diffractive Photoproduction of Dijets in $e p$ Collisions at HERA
}

\author{
Karel Černý * \\ Charles University in Prague - Institute of Particle and Nuclear Physics \\ V Holešovičkách 2, 18000 Prague 8 - Czech Republic
}

\begin{abstract}
Measurements are presented of differential dijet cross sections in diffractive photoproduction $\left(Q^{2}<0.01 \mathrm{GeV}^{2}\right)$ based on 1999 and 2000 HERA data with integrated luminosity of $54 \mathrm{pb}^{-1}$. The event topology is given by $e p \rightarrow e X Y$, where the system $X$, containing at least two jets, is separated from a leading low-mass proton dissociative system $Y$ by a large rapidity gap. The measurements are made in two kinematic ranges differing primarily in the transverse energy requirements on the two hardest jets. The dijet cross sections are compared with next-to-leading order QCD predictions based on recent diffractive parton densities obtained by H1. The next-to-leading order calculations predict larger cross sections than measured with the data. The cross section suppression of the data relative to the calculation is found to have no significant dependence on the photon four-momentum fraction entering the hard subprocess. There is a suggestion of a dependence of the suppression factor on the transverse energy of the jets.
\end{abstract}

In photoproduction two basic leading order (LO) classes of photon interactions can be distinguished - direct and resolved. In the direct processes the photon interacts as a pointlike particle. In the resolved processes the photon can develop its partonic structure and acts as a composite particle. In diffractive $e p$ scattering the proton stays intact or dissociates into a low-mass $\left(M_{Y} \ll E_{c m}\right)$ state. The hard diffractive processes can be considered as an exchange of a vacuum quantum number object (Pomeron, $\mathbb{P}$ ). A large gap in the rapidity distribution of the final state hadrons is observed. Owing to the photon structure there is an apparent resemblance between the resolved photoproduction and the hadron-hadron scattering. The factorization of the QCD calculable subprocess from the diffractive parton distribution functions (DPDF) in proton is not expected to hold in the hadron-hadron collisions [2]. The additional rescattering of spectators can fill the large rapidity gap and, therefore, to spoil the experimental signature of the diffractive event. Such a mechanism is expected to explain the difference between the measured structure function extracted from dijet production rates in $p \bar{p}$ collisions at Tevatron [3] and the theoretical predictions based on the diffractive parton densities by H1. Previous results concerning two jet production in diffractive photoproduction presented by the H1 and ZEUS collaborations can be found in [4] and [5], respectively. In [4] an overall suppression factor of 0.5 is applied to the NLO theory prediction in order to reproduce the measured cross section. In [5] a similar analysis is presented with somewhat higher $E_{T}$ range required on the jets. The global suppression factor of the NLO theory prediction is measured of 0.9 with the use of the same diffractive parton densities as in the case of [4]. The present measurements are based on luminosity of $54 \mathrm{pb}^{-1}$ which is about a factor of three larger than previous H1 measurement in a similar kinematic domain [4].

${ }^{*}$ On behalf of the H1 Collaboration. 


\section{Experimental Procedure}

A detailed description of the $\mathrm{H} 1$ detector can be found in [6]. The large rapidity gap method (LRG) is used for selection of the diffractive events which requires the forward detector instrumentation to be devoid of any activity above noise level.

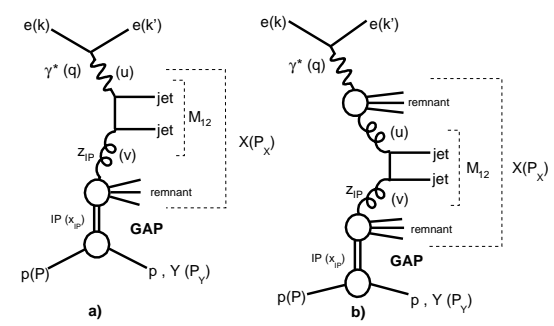

Figure 1: Leading order diagrams for diffractive dijet photoproduction at HERA for direct (a) and resolved (b) photon processes. The diffractive exchange is labeled with $\mathbb{P}$.

of kinematics used in [4] and [5].

The first measurement is performed in the kinematic range defined in (1)-(8) while the second measurement is modified according to (9)-(12), thus, the latter one matches the kinematic range of [5] except that it has a more restrictive $y$ and $Q^{2}$ ranges and different treatment of the proton dissociation contribution, i.e.

$$
\begin{aligned}
Q^{2} & <0.01 \mathrm{GeV}^{2}, \\
0.3 & <y<0.65, \\
E_{T}^{\text {jet } 1} & >5 \mathrm{GeV}, \\
E_{T}^{\text {jet } 2} & >4 \mathrm{GeV} \\
-1 & <\eta^{\text {jet } 1, \text { jet } 2}<2, \\
x_{\mathbb{P}} & <0.03
\end{aligned}
$$
two jets are shown, respectively.

In $[4,5]$ no dependence of the suppression factors is observed on the photon fourmomentum fraction $\left(x_{\gamma}\right)$ entering the hard subprocess. In both papers harder $E_{T}^{\text {jet1 }}$ differential cross section spectra are observed in the data than in the NLO theory prediction. This observation, though not very significant, indicates that there may be a dependence of the suppression factor on the $E_{T}$ range of the jets. Under these circumstances, the present analysis is performed in two cut schemes inspired by the ranges

In figures 1 (a) and (b) examples of the direct and resolved diffractive production of

where $E^{\text {jet } 1}$ and $\eta^{\text {jet } 1}$ are the transverse energy and pseudorapidity of the leading jet, respectively, both in the laboratory frame. The same notation applies to the sub-leading jet.

\section{Results and Conclusions}

Differential cross sections are measured for diffractive dijet photoproduction in two phase space regions with $\mathrm{H} 1$ data. The measured cross sections are compared with next-to-leading order QCD predictions obtained by two NLO QCD programs; Frixione et al. (FR) [7] and Klasen \& Kramer (KK) [8]. Three sets of diffractive parton distribution functions H1 2006 Fit A and Fit B [9] and H1 2007 Fit Jets [10] are used. 


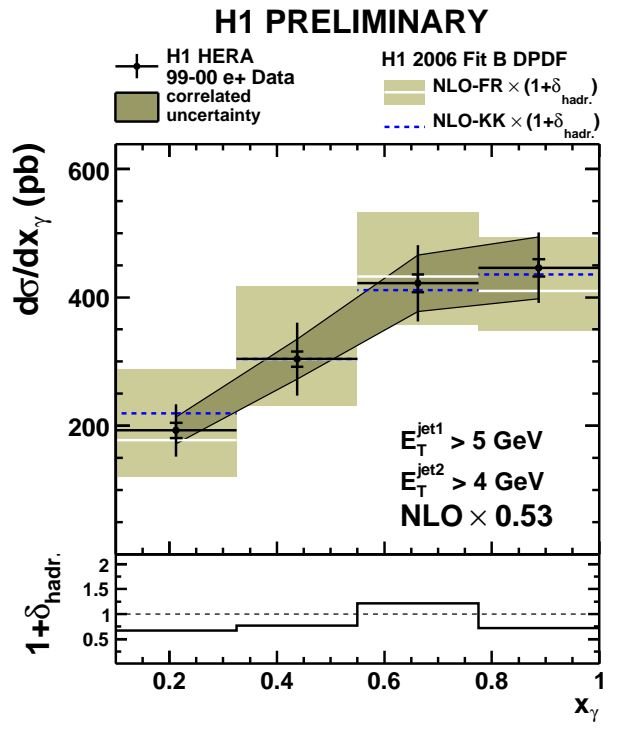

H1 PRELIMINARY

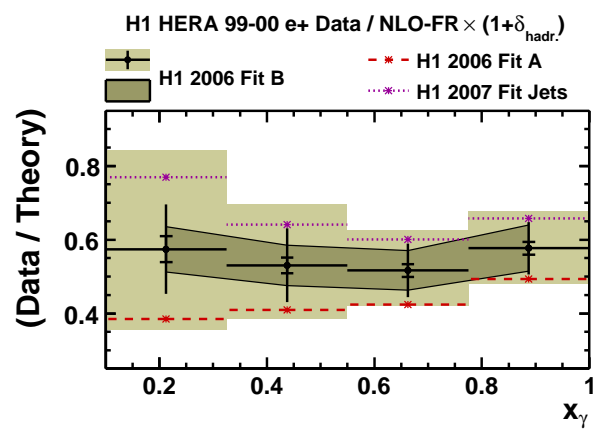

Figure 2: Result in lower $E_{T}$ range of jets. Upper plot: $d \sigma / d x_{\gamma}$ for data and NLO. Lower plot: ratio data/theory.
The results are presented as follows; in figure 2 the measurement is presented of $d \sigma / d x_{\gamma}$, where $x_{\gamma}$ is reconstructed as: $x_{\gamma}=\Sigma_{\text {jets }}(E-$ $\left.p_{z}\right) / \Sigma_{H F S}\left(E-p_{z}\right)$. The statistical and uncorrelated systematic uncertainties are added in quadrature and are represented with error bars (inner are statistical only). The correlated uncertainties are shown separately with a dark band. The FR and KK calculations are multiplied by the hadronization corrections (shown underneath) and by a common scale factor of 0.53. The NLO calculations are shown for the renormalization scale of $\mu_{r}=E_{T}^{\text {jet } 1}$. Predictions are also presented for FR Fit B with scale variations $\left(\mu_{r} / 2\right.$ and $\left.2 . \mu_{r}\right)$ depicted as a band around the central FR Fit B values. In the lower part of figure 2 ratios of $d \sigma / d x_{\gamma}$ measured in the data to the predictions are presented. No scaling factors are applied to the predictions in this case. The ratio is shown for the FR Fit B calculation with uncertainties propagated from the data. Also the renormalization scale variations of the ratio are presented for FR Fit B. Ratios of the data to FR Fit A and FR Fit Jets are also shown to approximate the effects of DPDF uncertainty. In a similar way the results have been studied for other variables.

In the analysis with lower $E_{T}$ cuts on the jets the FR NLO calculation based on Fit B overestimates the data. A global suppression factor $\left(S=\sigma^{\text {data }} / \sigma^{N L O}\right)$ is measured of $S^{(1)}=$ $0.54 \pm 0.01$ (stat.) \pm 0.10 (syst.) ${ }_{-0.13}^{+0.14}$ (scale.). The absence of any significant $x_{\gamma}$ dependence of the data/theory ratio (figure 2) and the value of $S^{(1)}$ are consistent with the previous $\mathrm{H} 1$ result [4]. The Fit B KK and FR calculations agree with each other independently. There is a suggestion of a dependence of the suppression factor on the $E_{T}$ of the jets. In figure 3 in the region of $E_{T}^{\text {jet } 1}<7 \mathrm{GeV}$ the suppression is in accordance with [4], a weakening one is suggested for the range of $E_{T}^{j e t 1}>7 \mathrm{GeV}$. Indeed, in the analysis with higher $E_{T}$ of the jets a weaker global suppression is measured for FR Fit B, $S^{(2)}=0.61 \pm 0.03$ (stat.) \pm 0.13 (syst.) ${ }_{-0.14}^{+0.16}$ (scale.). The absence of a significant $x_{\gamma}$ dependence of the data/theory ratio (figure 4 ) is consistent with the results in [5]. Taking all the uncertainties into account the suppression factor value $S^{(2)}$ is consistent with [5].

The presented results show that the NLO calculations overestimate the data globally (i.e. $x_{\gamma}$ independently), quantified by the suppression factors $S^{(1),(2)}$. The suggestion that the effect becomes weaker as $E_{T}$ of the jets increases is supported by the fact that $S^{(1)}<S^{(2)}$. 


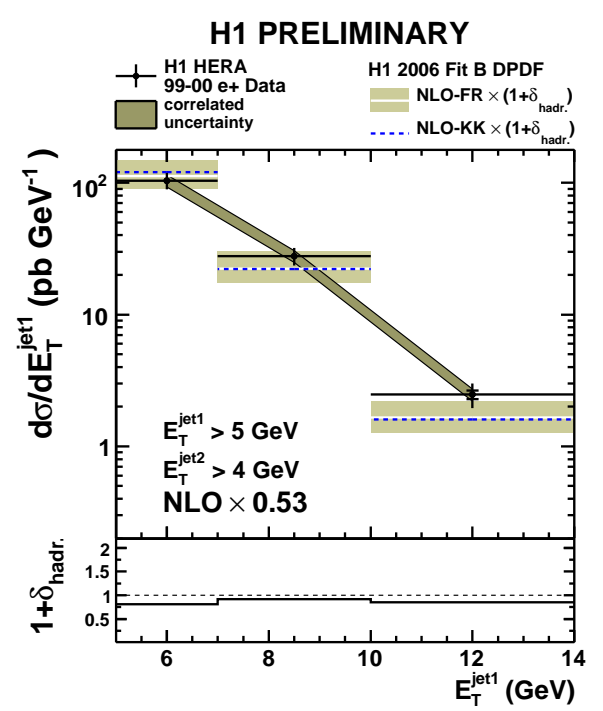

H1 PRELIMINARY

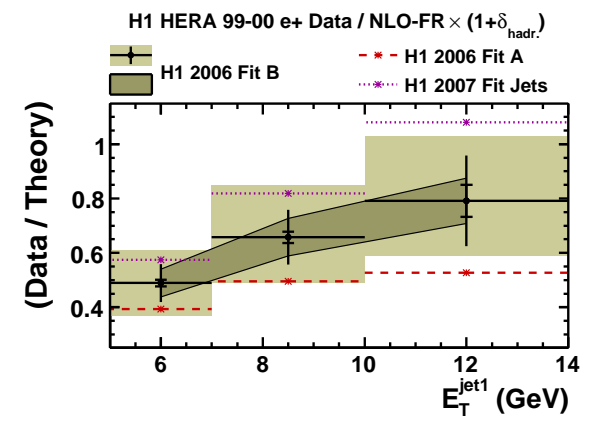

Figure 3: Result in lower $E_{T}$ range of jets. Upper plot: $d \sigma / d E_{T}^{\text {jet } 1}$ for data and NLO. Lower plot: ratio data/theory.

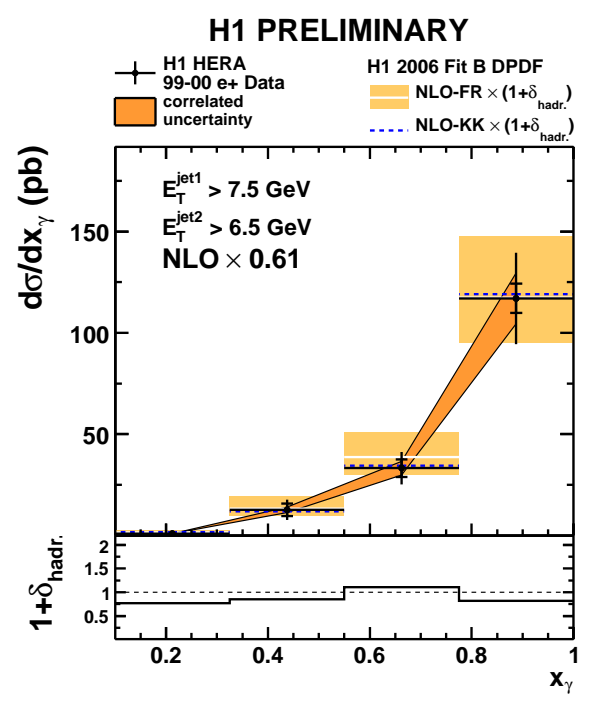

H1 PRELIMINARY

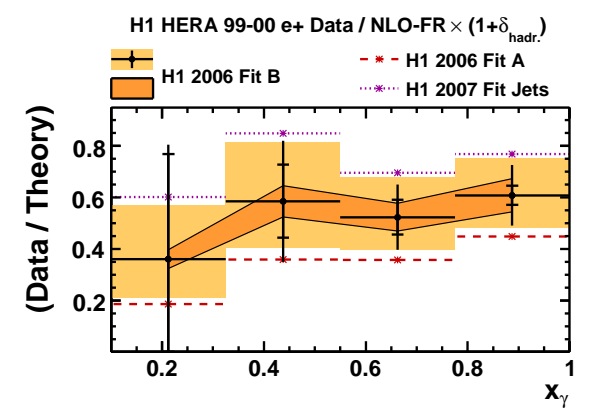

Figure 4: Result in higher $E_{T}$ range of jets. Upper plot: $d \sigma / d x_{\gamma}$ for data and NLO. Lower plot: ratio data/theory.

\section{References}

[1] Slides: http: //indico. cern. ch/materialDisplay $\cdot$ py? contribId=28\&sessionId=16\&material Id=slides\&conf Id=24657

[2] A.Kaidalov et al., Phys.Lett. B559 (2003) 235-238.

[3] T. Affolder et al. [CDF Collaboration], Phys. Rev. Lett. 84 (2000) 5043

[4] A. Aktas et al. [H1 Collaboration],Eur.Phys.J. C51 (2007) 549-568.

[5] S. Chekanov et al. [ZEUS Collaboration], submitted to Europ. Phys. Journal C. (2007).

[6] I. Abt et al. [H1 Collaboration], Nucl. Instrum. Methods A386 (1997) 310-347.

[7] S.Frixione, Z.Kunzst and A.Signer, Nucl. Phys. B467 (1996) 399 [hep-ph/9512328]; S.Frixione, Nucl. Phys. B507 (1997) 295 [hep-ph/9706545].

[8] M. Klasen and G. Kramer, Eur. Phys. J. C38 (2004.) 93.

[9] A. Aktas et al. [H1 Collaboration], Eur. Phys. J. C48 (2006) 715-748.

[10] A. Aktas et al. [H1 Collaboration], JHEP (2007) 0710:042. 